\title{
Slc11a2 is required for intestinal iron absorption and erythropoiesis but dispensable in placenta and liver
}

\author{
Hiromi Gunshin, ${ }^{1}$ Yuko Fujiwara, ${ }^{1,2}$ Angel O. Custodio, ${ }^{1}$ Cristina DiRenzo, ${ }^{1}$ \\ Sylvie Robine, ${ }^{3}$ and Nancy C. Andrews ${ }^{1,2}$ \\ ${ }^{1}$ Children's Hospital Boston, Dana-Farber Cancer Institute, Harvard Medical School, Boston, Massachusetts, USA. ${ }^{2 H o w a r d ~ H u g h e s ~ M e d i c a l ~ I n s t i t u t e, ~}$ \\ Boston, Massachusetts, USA. ${ }^{3}$ Equipe de Morphogenèse et Signalisation Cellulaires, Institut Curie, Paris, France.
}

\begin{abstract}
Solute carrier family 11, member 2 (SLC11A2) is the only transmembrane iron transporter known to be involved in cellular iron uptake. It is widely expressed and has been postulated to play important roles in intestinal iron absorption, erythroid iron utilization, hepatic iron accumulation, placental iron transfer, and other processes. Previous studies have suggested that other transporters might exist, but their physiological significance remained uncertain. To define the activities of Slc11a2 in vivo, we inactivated the murine gene that encodes it globally and selectively. We found that fetal Slc11a2 is not needed for materno-fetal iron transfer but that Slc11a2 activity is essential for intestinal non-heme iron absorption after birth. Slc11a2 is also required for normal hemoglobin production during the development of erythroid precursors. However, hepatocytes and most other cells must have an alternative, as-yet-unknown, iron uptake mechanism. We previously showed that Slc11a2 serves as the primary portal for intestinal iron entry in hemochromatosis. However, inactivation of murine $\mathrm{Hfe}$ ameliorates the phenotype of animals lacking Slc11a2.
\end{abstract}

\section{Introduction}

Iron is an essential nutrient that is toxic in excess. Intricate regulatory systems have evolved to preserve balance in an iron-rich environment. Homeostatic mechanisms ensure that tissue iron needs are met but not exceeded. Iron insufficiency can impair the function of iron-requiring enzymes in all tissues. However, anemia is the most apparent manifestation of iron deficiency because of the enormous amount of iron required for hemoglobin production in developing erythroid cells. There is no regulated excretion of iron through the liver or kidneys, and iron is lost from the body only through bleeding and sloughing of skin and mucosal cells. Surplus iron is generally stored in hepatocytes and macrophages. When tissue storage capacity is exceeded, unsequestered iron catalyzes the formation of damaging oxygen radicals, which leads to tissue fibrosis and clinical consequences of liver failure, cardiomyopathy, and endocrinopathies.

Initial iron stores are established through iron transfer from the mother to the developing fetus. After birth, iron is absorbed from food by epithelial cells (enterocytes) in the duodenum. It circulates in the bloodstream bound to transferrin and is delivered to sites of utilization and storage. Erythroid precursors, the primary consumers, are strictly dependent upon receptor-mediated endocytosis of transferrin through the transferrin cycle (1). Later, iron is recovered from old and damaged erythrocytes by tissue macrophages that phagocytose red blood cells and degrade hemoglobin to return the metal to the circulation.

Iron cannot pass through cellular membranes unassisted. To date, only 1 transmembrane transporter protein, solute carrier

Nonstandard abbreviations used: Hfe, hemochromatosis (gene); Slc11a2, solute carrier family 11, member 2 (gene); Trf, murine transferrin (gene); Trfr, murine transferrin receptor (gene).

Conflict of interest: The authors have declared that no conflict of interest exists.

Citation for this article: J. Clin. Invest. 115:1258-1266 (2005).

doi:10.1172/JCI200524356 family 11, member 2 (SLC11A2; also known as divalent metal ion transporter 1 [DMT1], Nramp2, and DCT1), is known to have physiological importance in bringing iron into cells. SLC11A2 acts as a proton-dependent iron importer of $\mathrm{Fe}^{2+}(2)$. It can also transport a variety of other divalent metal cations, including $\mathrm{Mn}^{2+}, \mathrm{Co}^{2+}, \mathrm{Cu}^{2+}$, and $\mathrm{Zn}^{2+}(2)$, though iron appears to be its most important physiological substrate. SLC11A2 is found on the apical membrane of duodenal enterocytes, consistent with its role in transepithelial iron transport (3). SLC11A2 is also found in transferrin cycle endosomes in erythroid precursors (4), where it may participate in transfer of iron to the cytoplasm. It is present in hepatocytes, where it has been postulated to be involved in non-transferrin-bound iron uptake (5). It is also expressed in the placenta, where it has been implicated in materno-fetal iron transfer $(6,7)$. Other iron uptake activities have been described in cultured cells, but their physiological significance and molecular identities are unknown (8-12).

Studies of animals carrying missense mutations in Slc11a2 previously suggested a major role for this transporter in intestinal nonheme iron transport and erythroid iron uptake. Microcytic anemia $(m k)$ mice and Belgrade (b) rats have systemic iron deficiency and anemia attributable to the same spontaneous missense mutation $(\mathrm{G} 185 \mathrm{R})$ in $\operatorname{Slc11a2}(13,14)$. The mutation impairs both subcellular localization (15) and transport activity $(16,17)$. We discovered that it also confers a novel calcium channel-like activity of uncertain importance (18). While studies on $m k$ mice and $b$ rats have provided insight into possible roles of Slc11a2 in vivo, the complexity of the partial loss-of-function mutation has made it difficult to draw firm conclusions.

A different missense mutation in SLC11A2 was described in a human patient who presented with anemia and hepatic iron overload (19). The mutation alters a splice junction, leading to substitution of aspartic acid for glutamic acid at residue 399 and preferential (but incomplete) skipping of exon 12. The 

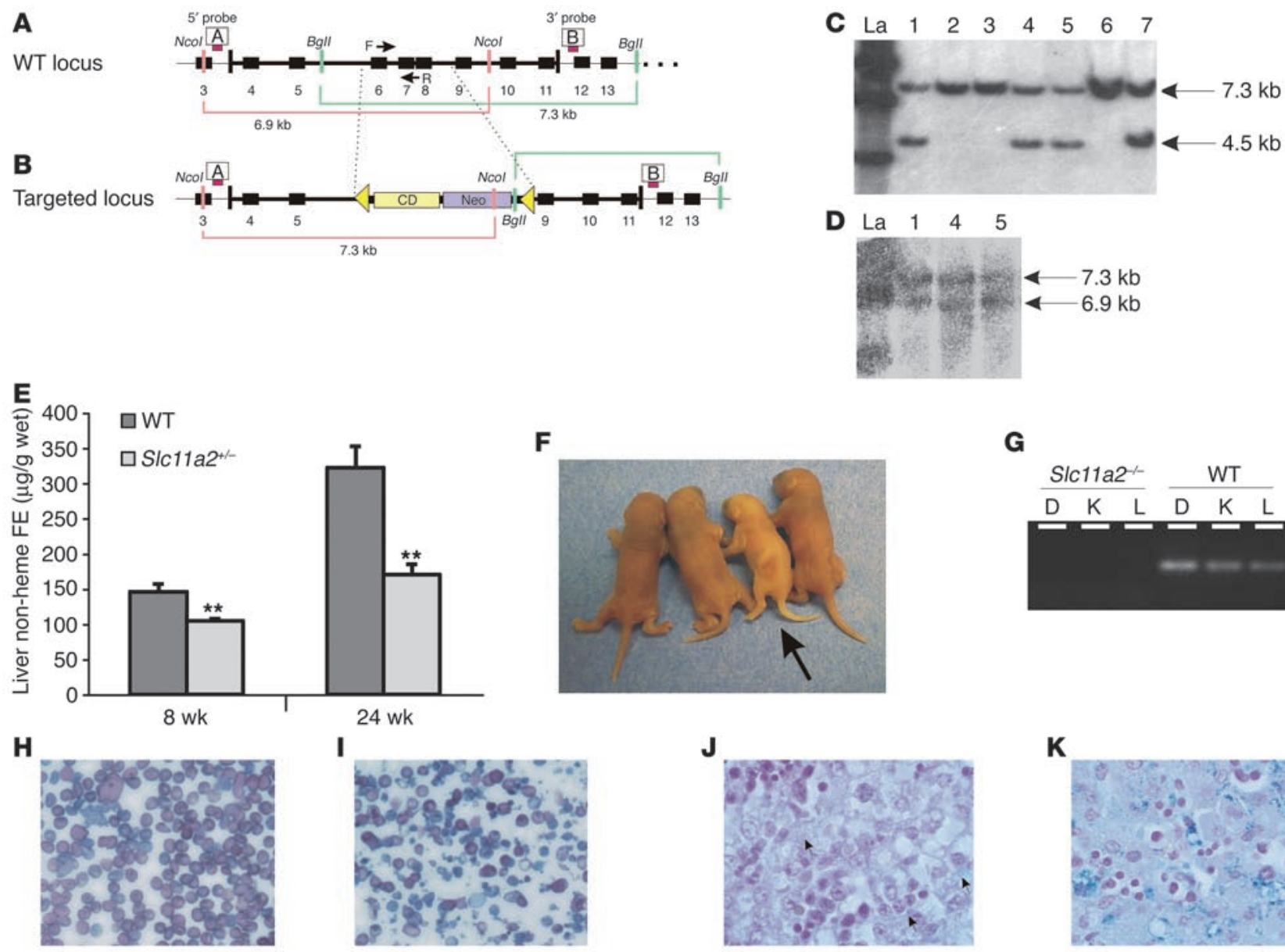

$\mathbf{F}$

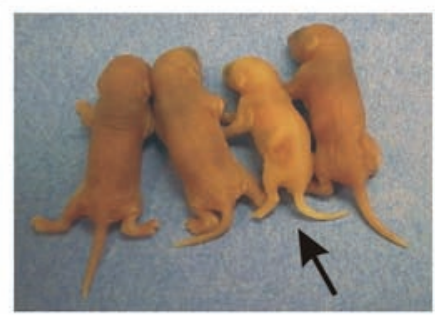

I

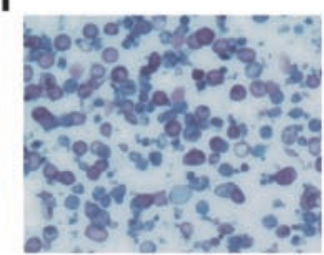

J

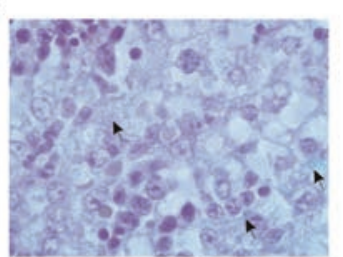

G

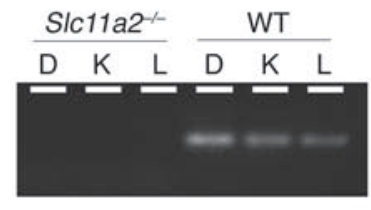

$\mathbf{K}$

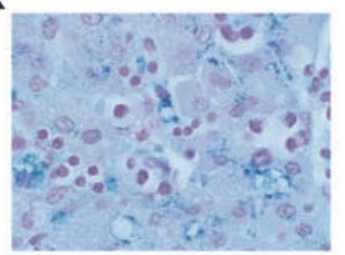

Figure 1

Targeted disruption of S/c11a2. S/c11a2 wild-type locus (A) and targeted locus (B). The targeting construct contained herpes simplex virus thymidine kinase gene (HSV-TK) and neomycin-resistance (Neo) and cytidine deaminase (CD) cassettes. Homologous recombination removed exons 6-8, replacing them with neomycin-resistance and cytidine deaminase cassettes flanked by loxP sites (triangles). The 5' and 3' probes used for Southern blot analysis are shown as red bars labeled A and B, respectively. F, forward; R, reverse. Southern blot analysis of clones using the $3^{\prime}$ probe (C) and the $5^{\prime}$ probe (D). Clones 1, 4, and 5 were correct at both ends. La, ladder. (E) Analysis of tissue iron content in female Slc11a2+/- and wild-type mice. (F) Wild-type and S/c11a2-/- (arrow) neonates on day 1 of life. Slc11a2 $2^{-/-}$mice were pale and runted. (G) PCR analysis of DNA prepared from duodenum (D), kidney $(\mathrm{K})$, and liver $(\mathrm{L})$ from S/c11a2 ${ }^{-/}$and wild-type mice using primer set $F$ and $R$ shown in $A$ and B. Morphology of peripheral blood smears from wild-type $(\mathbf{H})$ and S/c11a2 ${ }^{--}$(I) mice. Original magnification, $\times 40$. The mutant mice had hypochromic, microcytic cells with marked anisocytosis and poikilocytosis. Perls Prussian blue iron staining of wild-type neonatal liver (J) and S/c11a2 ${ }^{-/-}$neonatal liver $(\mathbf{K})$. Original magnification, $\times 100$. There was little iron deposition in hepatocytes and Kupffer cells (arrows) of wild-type mice but marked iron deposition in those of S/c11a2-/- mice following a single intraperitoneal injection of iron dextran (5 mg iron).

improperly spliced mRNA retains the correct reading frame but codes for a protein lacking one of its transmembrane segments. The correctly spliced mRNA encodes a variant of SLC11A2 that retains iron transport capability, but the incorrectly spliced mRNA does not (H. Gunshin, J. Jin, Y. Fujiwara, and N.C. Andrews, unpublished observations). The patient's phenotype suggests that SLC11A2 function is impaired in erythroid cells but that intestinal absorption and hepatic iron uptake remain intact. Incomplete information on the tissue expression of the 2 spliced forms makes it difficult to draw conclusions about the normal roles of SLC11A2 in human subjects.

The purpose of our study was to clarify which cell types are dependent upon Slc11a2 function and to determine whether other transmembrane iron import pathways are physiologically significant. We anticipate that our findings will be relevant to human physiology because there are strong similarities between murine and human iron metabolism (20). We inactivated the murine Slc11a2 gene globally and in selected tissues through gene targeting and homologous recombination in pluripotential embryonic stem cells.

Global inactivation of Slc11a2 resulted in a phenotype that is more severe than that seen in animals homozygous for the G185R mutation. We found that fetal Slc11a2 is not needed for materno-fetal iron transfer but is necessary for normal iron uptake by erythroid precursor cells and the intestine. We establish that at least 1 efficient, alternative iron uptake pathway must be active in the placenta, in hepatocytes, and possibly in other somatic cell types. Surprisingly, we found that mice with genetic hemochromatosis can better tolerate loss of Slc11a2, in spite of the fact that most iron loading in hemochromatosis appears to involve Slc11a2-mediated iron transport. 


\section{Table 1}

Phenotype of Slc11a2-- mice

\begin{tabular}{|c|c|c|c|}
\hline & $\begin{array}{l}\text { WT }(n=8) \\
( \pm \text { SEM) }\end{array}$ & $\begin{array}{c}\text { SIc11a2- }(n=16) \\
( \pm \text { SEM })\end{array}$ & $P$ values \\
\hline Body wt (g) & $2.02 \pm 0.25$ & $1.34 \pm 0.05$ & $P<0.0001$ \\
\hline Liver Fe $(\mu \mathrm{g} / \mathrm{g})$ & $87.30 \pm 4.66$ & $269.69 \pm 12.85$ & $P<0.0001$ \\
\hline Spleen Fe $(\mu \mathrm{g} / \mathrm{g})$ & $102.74 \pm 5.80$ & $90.82 \pm 8.63$ & NS \\
\hline Hemoglobin (g/dl) & $10.83 \pm 0.65$ & $4.33 \pm 0.45$ & $P<0.0001$ \\
\hline Hematocrit (\%) & $33.83 \pm 1.23$ & $15.08 \pm 0.86$ & $P<0.0001$ \\
\hline rbcs (1012 cells/l) & $3.49 \pm 0.14$ & $2.40 \pm 0.13$ & $P<0.0001$ \\
\hline $\begin{array}{r}\text { Mean erythrocyte } \\
\text { cell volume (fl) }\end{array}$ & $95.06 \pm 1.64$ & $62.68 \pm 1.00$ & $P<0.0001$ \\
\hline
\end{tabular}

Measurements are given for 3-day-old wild-type and S/c11a2-- mice. Tissue iron content measurements were performed with wet tissue.

\section{Results}

Targeted disruption of Slc11a2. The murine Slc11a2 gene consists of 18 exons spread over more than $30 \mathrm{~kb}$ on distal chromosome 15 . The G185R mutation lies in the fourth of 12 predicted transmembrane segments. We designed a targeting construct to delete exons 6-8, corresponding to predicted transmembrane segments 3-5 (Figure 1, A and B). This construct was introduced into mouse ES cells by electroporation. Correct homologous recombination was confirmed by Southern blot analysis of both ends of the recombined segment (Figure 1, C and D). The targeted allele was introduced into the germline and maintained on a homogeneous 129 S6/SvEvTac inbred background.

Slc1 $1 \mathrm{a} 2^{+/-}$mice appeared normal, and there were no significant abnormalities in their hematological parameters (data not shown). However, by 8 weeks of age, their liver iron content was lower than that of wild-type mice (Figure 1E), which indicates a mild effect of haploinsufficiency. This could result from a mild impairment of intestinal iron absorption, a mild defect in hepatocyte iron assimilation, or both.

Slc11a2-/- mice were born alive. Although there were no apparent anatomical abnormalities, they were noticeably pale at birth (Figure 1F). Inactivation of Slc11a2 was confirmed by PCR analysis (Figure 1G). Slc11a2 $2^{-/-}$mice showed progressive postnatal growth retardation. By postnatal day 3 (P3), their body weights were $30 \%$ less than those of wild-type (Table 1) and Slc11a2+littermates (data not shown). None survived more than 7 days. Peripheral blood smears from Slc11a2-/- pups showed hypochromic and microcytic erythrocytes with marked anisocytosis and poikilocytosis, characteristic of severe iron-deficiency anemia (Figure 1I). Accordingly, analysis of blood at P3 showed decreased hemoglobin concentration, hematocrit, red blood cell number, and mean erythrocyte cell volume (Table 1). All differences were highly significant. We confirmed that the phenotype was not attributable to residual vector sequences in the Slc11a2 locus by analyzing animals in which the targeting cassette was deleted by Cre recombinase (data not shown).

However, Slc11a2-/- mice were not iron deficient in all tissues. Total body iron content, normalized for body weight, was similar in Slc11a2 $2^{-/-}$mice and controls, which indicates that placental iron transfer was efficient (data not shown). Liver iron stores

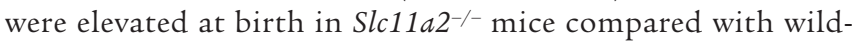
type littermates (Table 1 ), which suggests redistribution of iron from the erythron to the liver. To ensure that abundant iron was available for erythropoiesis, we administered iron dextran by injection. This markedly increased liver iron stores in both hepatocytes and macrophages (Figure $1 \mathrm{~K}$ ). However, it did not prolong survival of any Slc11a2 $2^{-/}$pups (data not shown). In contrast, rbc transfusions allowed approximately $5 \%$ of the Slc11a2-/mice to survive until weaning, which suggests that anemia was the likely cause of death.

A role for Slc11a2 in bematopoietic cells. Based on these findings and those of earlier studies (14), we suspected that Slc11a2 plays an important role in iron acquisition by erythroid precursors. To address this directly, we isolated fetal liver HSCs from Slc11a2-/and control animals and transplanted them into lethally irradiated wild-type mice.

We examined hematopoietic reconstitution by analysis of hemoglobin variants and flow cytometry. The recipient mice had a mixed C57BL/6 and 129S6/SvEvTac background. The donor mice had a homogeneous 129S6/SvEvTac background. We observed that the hemoglobin from transplant recipients was predominantly of 129S6/SvEvTac origin (Figure 2A). Little, if any, C57BL/ 6 hemoglobin was detectable. Accordingly, flow cytometry analysis confirmed that nearly $100 \%$ of the circulating blood cells carried only the CD45.2 surface marker, which indicates that they were of 129S6/SvEvTac origin (Figure 2B). These results demonstrate that the recipients had been successfully reconstituted with the transplanted cells.

As expected, peripheral blood smears from animals transplanted with wild-type HSCs were normal (Figure 2C). In contrast, blood smears from Slc11a2 ${ }^{-/-}$HSC recipients showed abnormal erythrocyte morphology consistent with iron-deficient erythropoiesis (Figure 2D). Eight weeks after transplantation, hemoglobin levels were substantially lower in mice that had received Slc11a2-/- HSCs. This anemia persisted, while normal hemoglobin levels were maintained in control recipients (Figure 2E).

We expected nonhematopoietic tissues in recipients of Slc11a2-/HSCs to express Slc11a2 normally, because they were of wildtype origin. However, we found that Slc11a2-/- HSC recipients progressively accumulated excess iron in the liver (Figure 2F). This suggests either a compensatory increase in intestinal iron absorption or a redistribution of iron that was not effectively utilized by erythroid cells.

Slc11a2 is important for normal intestinal iron absorption. To avoid early lethality due to total loss of Slc11a2, we developed mice carrying an Slc11a2 allele that had loxP recombination sites flanking exons 6-8 of the Slc11a2 gene but no other residual vector sequences (Figure $3, \mathrm{~A}-\mathrm{F}$ ). Insertion of loxP sites into the floxed allele left the Slc11a2 gene intact, allowing for normal expression. We confirmed that mice homozygous for this allele, referred to as Slc11a2flox/flox, were phenotypically normal (data not shown). When these mice were bred with mice expressing Cre recombinase, the Slc11a2 gene was selectively inactivated wherever Cre was present.

To inactivate Slc11a2 in the intestinal epithelium, we bred Slc11a flox/flox mice with mice carrying a Villin-Cre transgene, which allowed for Cre expression under the control of the intestinespecific villin promoter. Previous studies have shown that in Villin-Cre mice, Cre/loxP recombination is mediated exclusively and homogeneously in the extraembryonic visceral endoderm in utero and in intestinal epithelial cells before and after birth (21). Viable mice were born from this cross, which indicates that loss of Slc11a2 in the extraembryonic visceral endoderm was not 
A

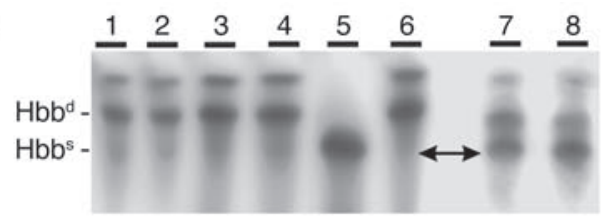

B

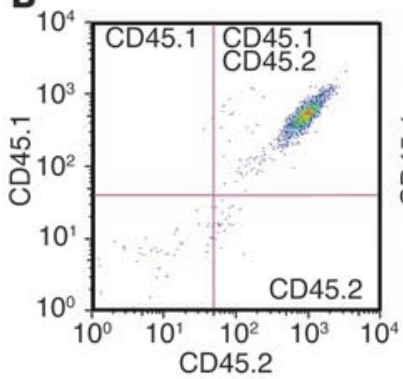

C

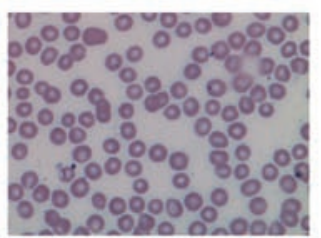

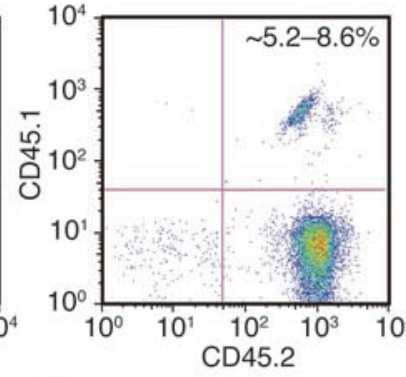

D

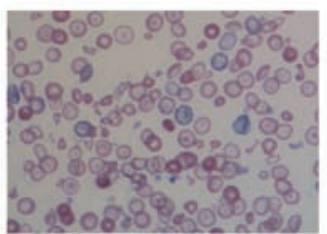

E

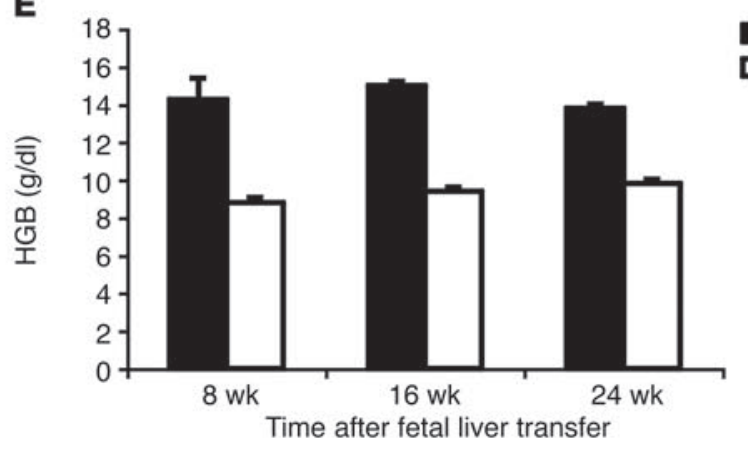

$\mathbf{F}$

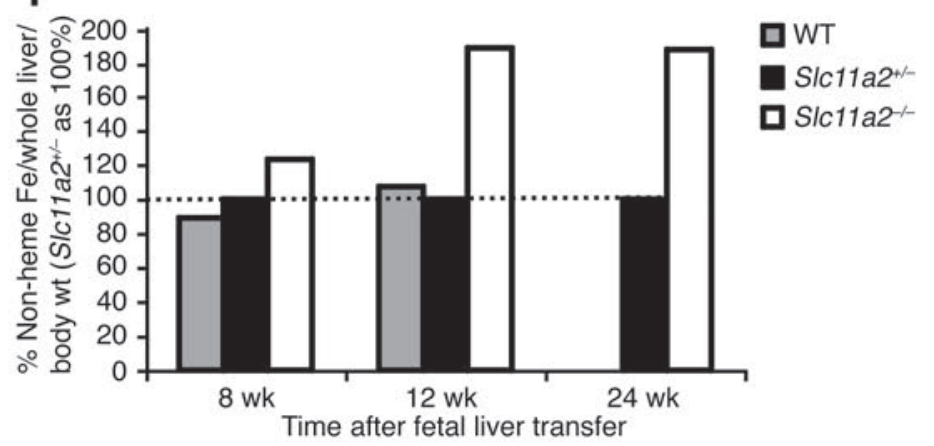

Figure 2

Hematopoietic Slc11a2 is required for normal erythropoiesis. (A) Hemoglobin isoform analysis demonstrating the presence of HSC-derived hemoglobin in the peripheral blood 8 weeks after fetal liver transfer. The blood samples were as follows: lanes 1 and 2, S/c11a2-/- HSC recipients; lanes 3 and 4, wild-type HSC recipients; lane 5, C57BL/6 mice; lane 6, 129S6/SvEvTac mice; lanes 7 and 8, recipients before transfer. After fetal liver transfer, recipients produced diffuse alleles of hemoglobin $\beta$ chain $\left(\mathrm{Hbb}^{\mathrm{d}}\right.$ ) characteristic of the 129S6/SvEvTac background but not single alleles of $\mathrm{Hbb}\left(\mathrm{Hbb}^{\mathrm{s}}\right)$ characteristic of the C57BL/6 background. (B) Flow cytometry of peripheral blood before (left) and 8 weeks after (right) fetal liver transfer. Before HSC transfer, 100\% of recipient cells had both CD45.1 and CD45.2 markers characteristic of their mixed C57BL/6 and 129S6/SvEvTac background (left). After fetal liver transfer, hematopoietic cells primarily expressed only the CD45.2 marker associated with the 129S6/SvEvTac background, which was consistent with the hemoglobin analysis. Approximately $5.2-8.6 \%$ of cells were double stained for CD45.1 and CD45.2 markers, which indicates a low level of residual chimerism. Wild-type blood morphology was normal (C) and distinctly different from that of recipients of S/c11a2-- HSCs (D). Original magnification, $\times 60$. The recipients of S/c 11a2 ${ }^{-1-} \mathrm{HSCs}^{-1}$ had hypochromic, microcytic cells with anisocytosis and poikilocytosis. (E) Hemoglobin (HGB) levels were persistently lower in S/c11a2 ${ }^{-/-}$HSC recipients compared with animals that received Slc11a2+/- HSCs. (F) Non-heme liver iron content (adjusted taking into account whole liver and body weight) was increased in animals that received S/c11a2 ${ }^{-/-}$HSCs. Values are shown as a percent of the values determined for S/c11a2 ${ }^{+/-}$HSC recipients.

deleterious. They will be referred to as Slc11a2 $2^{\text {int } / \text { int }}$ mice. We evaluated Slc11a2 expression on immunoblots prepared from isolated enterocytes. We had previously observed that hypotransferrinemic (transferrin ${ }^{h p x / h p x}\left[\operatorname{Tr} f^{h p x / h p x}\right]$ ) mice expressed high levels of intestinal Slc11a2 (22), and we used $\operatorname{Tr} f p p x / h p x$ as a positive control in the present study. We were unable to detect Slc11a2 in samples

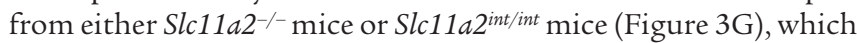
supports the conclusion that the floxed allele had been efficiently excised in enterocytes. Southern blot analysis confirmed that the allele was excised in the intestine but not in other postnatal tissues (Figure $3 \mathrm{H}$ ). The unexcised, floxed allele was also present in DNA prepared from whole intestinal segments, likely due to contamination by other cell types that do not express villin (e.g., connective tissue, muscle, blood).

No abnormalities were observed in neonatal hematological parameters or tissue iron content (data not shown). However, after birth, when they became dependent upon intestinal nutrition, the Slc11a2 int/int mice developed progressive anemia (Figure 4A). While this anemia was profound, it was never as severe as that seen in Slc11a2-/- mice.
Significant growth retardation was apparent in Slc11a $2^{\text {int/int }}$ mice by 12 weeks of age (Figure 4B). Non-heme tissue iron content was diminished in the liver (Figure 4C), spleen (Figure 4D), kidney, brain, and heart (Figure 4E) of both Slc11a2-/- and Slc11a2 int/int mutants. Both mutants displayed marked splenomegaly and cardiomegaly (data not shown). Histological analysis (data not shown) revealed markedly expanded red pulp with dysplastic erythropoiesis and greatly increased rate of apoptosis in the mutant spleens. There was prominent extramedullary erythropoiesis in the liver, particularly in sinusoidal regions. These abnormalities are likely attributable to severe anemia. When iron dextran was administered to bypass the apparent intestinal iron absorption defect in Slc11a2 int/int mice, their anemia resolved (data not shown), which confirms that iron delivery to other tissues was not impaired.

We compared the survival of singly transfused Slc11a2-/- mice and untreated Slc11a2 int/int mice. Both strains had poor overall survival, but Slc11a2-/- mice died significantly earlier (Figure 4F). The less severe phenotype and longer survival of Slc11a2 int/int mice could be explained by normal Slc11a2 activity in other cell types, particularly in erythroid precursors. 

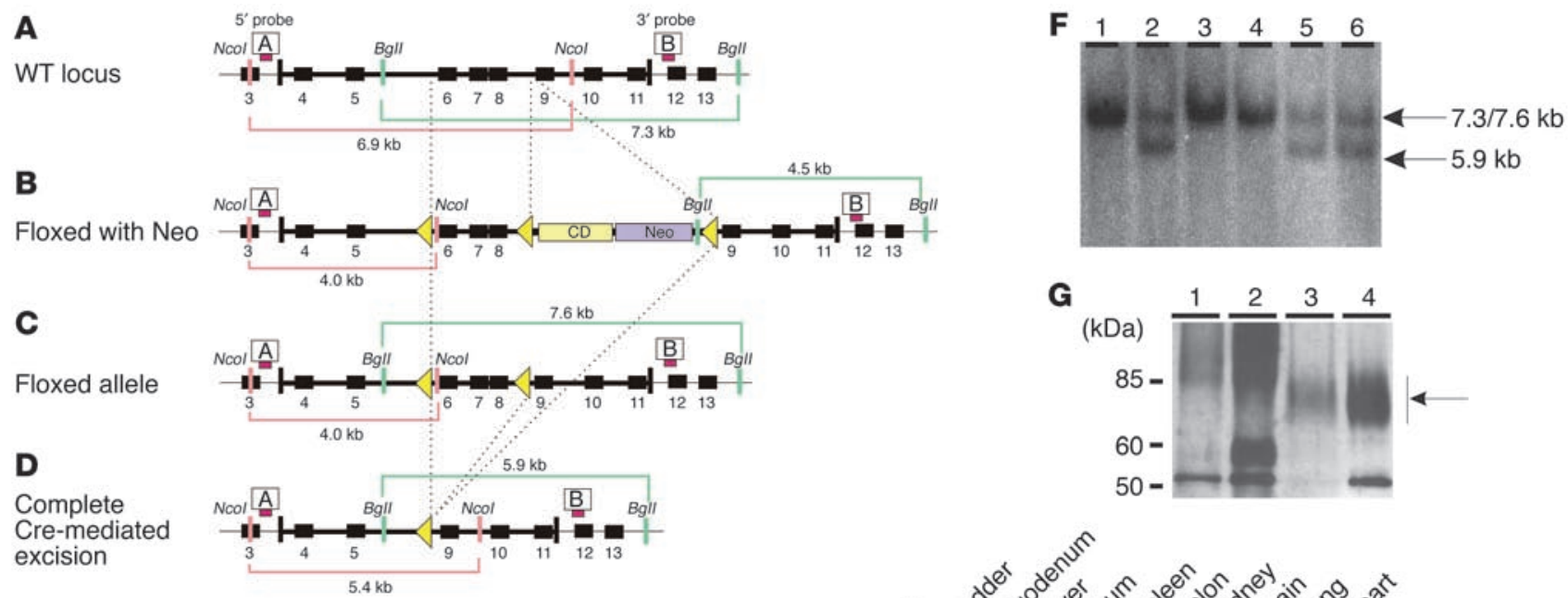

G
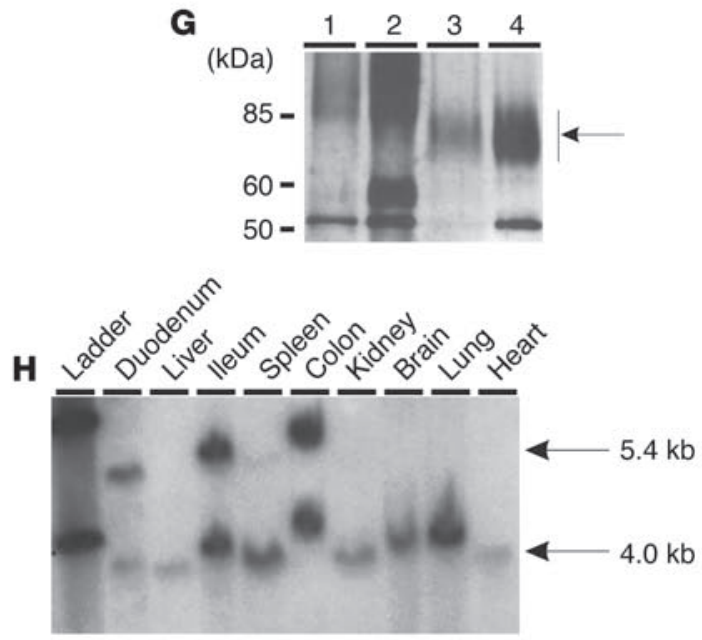

Figure 3

Conditional inactivation of Slc11a2 in the intestine. Slc11a2 wild-type locus (A) and targeted locus (B) after introduction of an intronic floxed neomycin resistance and cytosine deaminase cassettes and an additional loxP site between exons 5 and 6 . Positions of $5^{\prime}$ and $3^{\prime}$ probes used for Southern blot analysis are shown as red bars labeled A and B, respectively. (C) Partial Cre recombinase-mediated excision in which the Neo and cytosine deaminase cassettes were removed but the coding sequence was left intact. (D) S/c11a2 locus after complete excision to inactivate the gene. (E) Southern blot analysis of the $5^{\prime}$ end of the locus in ES cells after Cre transfection. (F) Southern blot analysis of the $3^{\prime}$ end of the locus in ES cells after Cre transfection. Clone 3 retained the floxed allele and was used to generate S/c11a2flox/flox mice; clones 2, 5, and 6 underwent complete deletion and were used to generate S/c11a2-/- mice without residual Neo and cytosine deaminase cassettes. (G) Immunoblot analysis of duodenum from Slc11a2 ${ }^{-/-}$(lane 1), Slc11a2 intlint (lane 2), wild-type (lane 3) and Trfhpx/hpx (lane 4) mice. Protein amounts loaded per gel were 4 $\mu \mathrm{g}$ of S/c11a2--1, $2 \mu \mathrm{g}$ of S/c11a2 intlint, $50 \mu \mathrm{g}$ of wild-type, and $2 \mu \mathrm{g}$ of Trfhpx/hpx duodenal lysates. Slc11a2 migrates as a diffuse band between the $60-\mathrm{kDa}$ and $85-\mathrm{kDa}$ markers (arrow). (H) Southern blot analysis of S/c11a2intlint mice resulting from an intercross of S/c11a2 flox/flox and Villin-Cre mice using probe $\mathrm{A}$, demonstrating that the deletion of the floxed region occurred only in intestinal tissues.

Mice homozygous for the mk mutation have residual iron transport activity. Mice homozygous for a G185R missense mutation in Slc11a2 (13) were originally propagated on a novel inbred strain, $\mathrm{MK} / \mathrm{ReJ}(23)$. On that background, fewer than $10 \%$ of Slc11 $2^{\mathrm{mk} / \mathrm{mk}}$ homozygous mice survived to weaning (24). To directly compare

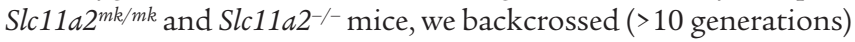
the G185R mutation onto the same 129S6/SvEvTac background present in our Slc11a2 $2^{-/-}$animals.

In this context, nearly $100 \%$ of the Slc1 $1 \mathrm{a} 2^{\mathrm{mk} / \mathrm{mk}}$ mice survived to adulthood. This survival was substantially better than that seen in Slc11a2-/- mice. Liver non-heme iron content was significantly

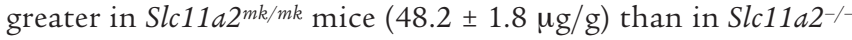
mice $(26.5 \pm 1.0 \mu \mathrm{g} / \mathrm{g} ; P<0.0001)$. These results confirm that the G185R Slc11a2 protein retains significant iron transport activity in vivo. Furthermore, they indicate that other genetic factors, differing between the MK/ReJ and 129S6/SvEvTac strains, act as modifiers of iron homeostasis.

Hfe deficiency ameliorates the Slc11a2 ${ }^{-/-}$phenotype. We previously showed that Slc11a $2^{m k / m k}$ mice fail to develop iron overload when the disease-related gene hemochromatosis ( $\mathrm{Hfe}$ ) is inactivated (25). That result suggested that iron overload in HFE hemochromatosis involves increased iron transport through a pathway involv- ing Slc11a2. In an attempt to confirm that result, we crossed

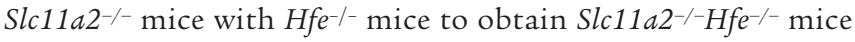
carrying mutations in both genes.

We were surprised to find that survival of Slc11a2 $12^{-/-}$mice was significantly improved when only $1 \mathrm{Hfe}$ allele was inactivated (data not shown). This effect was more pronounced in Slc11a2-/$\mathrm{Hfe}^{-/-}$animals. At 12 weeks of age, body weight and liver iron con-

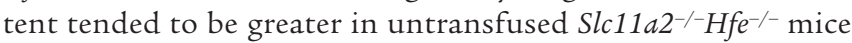
than in Slc11a2 $2^{-/-}$mice that had received a single rbc transfusion. The hematocrits of compound mutant mice were also significantly higher than those of singly transfused Slc11 a2 $2^{-/-}$mice lack-

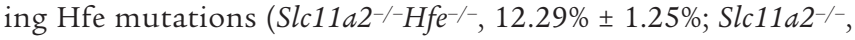
$6.10 \% \pm 1.60 \%, P=0.0186)$. A similar effect was observed in Slc11a2 $2^{m / m k} H f e^{-/-}$mice (data not shown).

\section{Discussion}

Previous studies of animals carrying a G185R missense mutation in Slc11a2 suggested that the protein was important for both intestinal iron absorption and erythroid iron utilization $(13,14)$. However, those studies were limited for 2 reasons. First, the missense mutation did not result in total loss of protein function. Second, we discovered that the mutation conferred a new calcium 
A

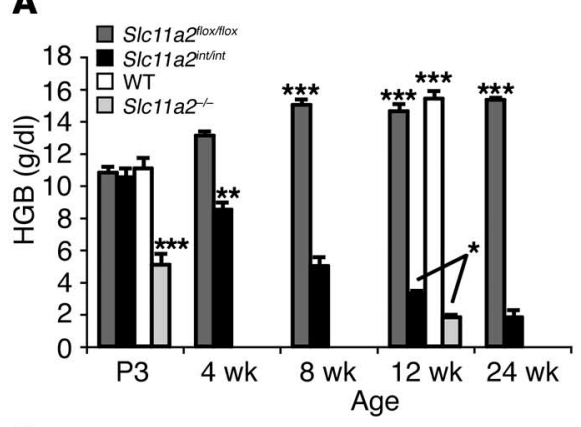

D

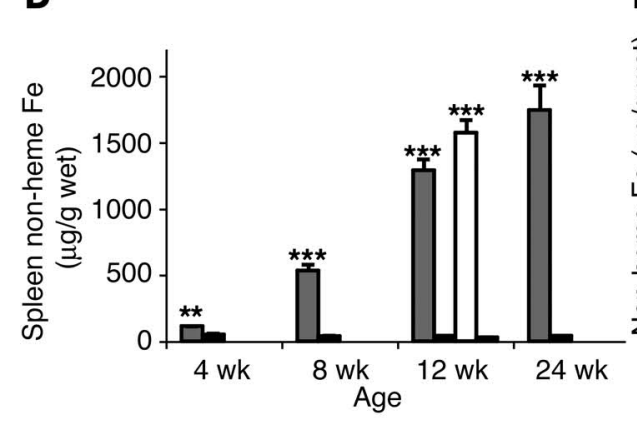

B

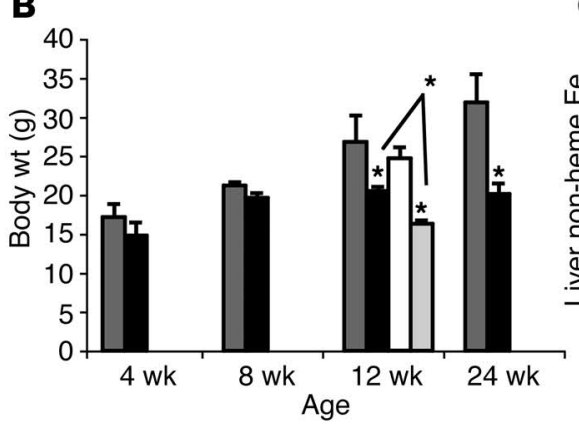

E

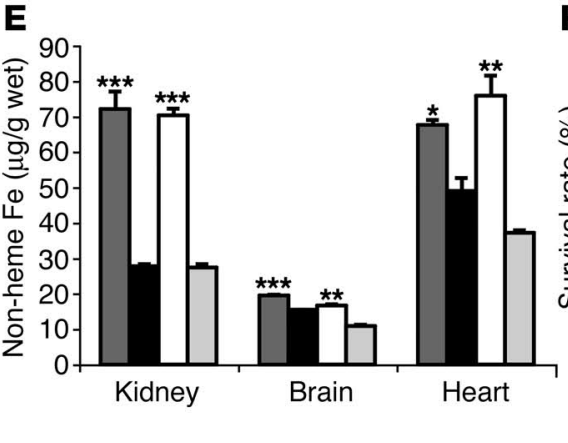

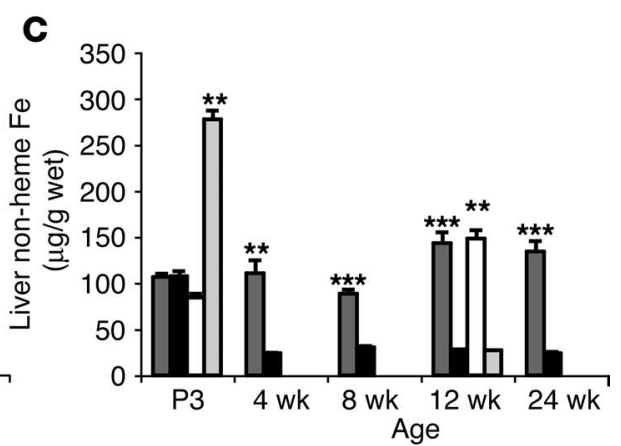

$\mathbf{F}$

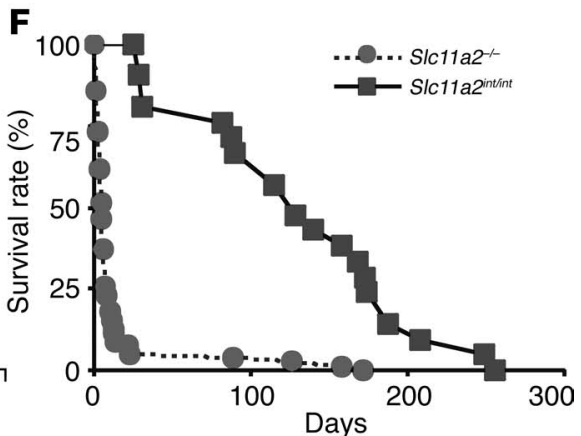

Figure 4

Liver and spleen iron loading in mice with Slc11a2 mutations. (A) Hemoglobin levels. (B) Body weights. (C and D) Tissue non-heme iron content at ages 3 days (P3) to 24 weeks (E) Tissue non-heme iron content at age 12-14 weeks. Results in micrograms/gram wet weight are expressed as mean \pm SEM $(n=4-16)$. Statistical analysis was performed using the unpaired Student's $t$ test, comparing mutant and wild-type mice: ${ }^{\star} P<0.05 ;{ }^{* *} P<0.005 ;{ }^{* *} P<0.0001$. (F) Survival of mutant mice. S/c11a2-/- neonates were transfused once with rbcs on the first day of life. S/c11a2 intlint mice were not transfused.

channel activity of uncertain significance (18). To more definitively assess the role of Slc11a2 in normal iron homeostasis, we have developed mouse models in which Slc11a2 is inactivated both globally and in selected tissues.

Slc11a2 $2^{-1-}$ mice are born anemic but without developmental abnormalities. We can conclude that fetal Slc11a2 is not essential before birth and is not necessary for the proliferation or differentiation of most cell types. Indeed, the neonatal iron endowment was normal, and liver iron stores were increased at birth, which indicates that materno-fetal iron transfer was efficient in the absence of Slc11a2. Although Slc11a2 is normally found in placenta, its role must not be essential. Another transporter must be responsible for iron uptake by the fetal-derived cells that effect materno-fetal iron transfer.

Our results further indicated that Slc11a2 was unnecessary for fetal hepatocyte iron accumulation. When we administered iron dextran to newborn animals, we observed dramatic accumulation of iron in both macrophages and hepatocytes of the liver. This observation does not, in itself, rule out a role for Slc11a2 in normal macrophage iron uptake. Macrophages internalize iron dextran by phagocytosis. Iron accumulates at high levels, but its intracellular metabolism has not been characterized in detail. Hepatocytes are not known to phagocytose iron dextran; it is likely, but unproven, that they acquire iron that has already been processed by and released from macrophages. Selective inactivation of a floxed Slc11a2 allele by liver-specific expression of Cre recombinase partially, but incompletely, interrupted hepatocyte iron accumulation (H. Gunshin and N.C. Andrews, unpublished observations).

Despite adequate stores, Slc11 a2 $2^{-/}$animals were unable to use iron efficiently for erythropoiesis. They were severely anemic from birth, and anemia persisted even after administration of iron dextran. The anemia was the likely cause of postnatal death in the first week of life. However, the defect was not absolute - partially hemoglobinized erythrocytes were present, though iron deficient.

We transplanted Slc11a2-/- HSCs into irradiated wild-type recipients. Nearly all circulating blood cells were derived from the Slc11a2-/- HSCs. Although Slc11a2 was expressed normally in nonhematopoietic tissues, the transplanted animals developed frank iron-deficiency anemia. This confirms the importance of Slc11a2 in erythroid iron uptake. Formally, our HSC transfer experiments indicate only that expression of Slc11a2 in some hematopoietic cell type is necessary for normal erythropoiesis. However, we believe that it is unlikely that Slc11a2 carries out its erythropoietic function in macrophages or other nonerythroid cells.

An alternative erythroid iron uptake system could involve direct transfer of iron across the plasma membrane, function of a different iron transporter in transferrin cycle endosomes, or both. We previously found that mice lacking a functional transferrin receptor gene ( $\operatorname{Trfr}^{-1-}$ mice) died during intrauterine devel-

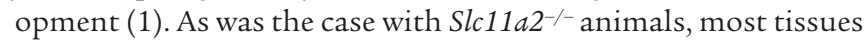
developed normally, and erythroid cells were present. However, prenatal anemia appeared to be the cause of fetal death. If that is correct, then the anemia was more severe in $\mathrm{Trfr}^{-/}$than in Slc11a2 $2^{-/}$mice, which suggests that loss of the transferrin cycle has more deleterious consequences for erythropoiesis than does loss of Slc11a2. Furthermore, Trfr ${ }^{-/-}$embryos showed an increased rate of apoptosis of primitive neuroepithelial cells (1), and $\mathrm{Trfr}^{-1-}$ ES cells showed defects in lymphopoietic differentiation (26). Although we did not investigate the nervous system 


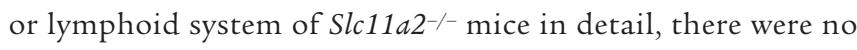
overt abnormalities. Taken together, these results suggest that Slc11a2 may not be the sole iron transporter functioning in transferrin cycle endosomes.

Slc11a2 was originally identified as a candidate for the apical enterocyte iron transporter in the intestine $(2,13)$. We wanted to formally test this hypothesis in Slc11a2 ${ }^{-/-}$mice, particularly in light of a description of a human patient with a mutation in SLC11A2 who had adequate intestinal iron absorption (19). However, it was difficult to ascertain the role of Slc11a2 in iron absorption in Slc11a2-/- mice due to the severity of the anemia and the fact that it was manifest before birth. To circumvent this problem, we developed a floxed Slc11a2 allele and selectively disrupted the gene in the intestine. While we cannot be certain that all enterocytes lacked Slc11a2, we could not detect the protein on immunoblots prepared from duodenal epithelial cells. The phenotype of the Slc11a2 int/int animals was severe. As expected, they were normal at birth, but their prenatal iron stores rapidly depleted, and they developed iron-deficiency anemia. These results confirm that Slc11a2 is the major intestinal iron transporter acting to take up non-heme iron from the diet.

We have not addressed whether Slc11a2 is important for heme iron absorption. Heme iron is thought to bind to a cell surface heme receptor on enterocytes (27), but it remains unclear how it enters the cytoplasm. We speculate that the heme-heme receptor complex may be internalized in a manner analogous to the transferrin cycle, to allow for enzymatic liberation of iron from heme within an intracellular compartment. There is no direct evidence supporting or refuting this model at present, but it is intriguing that others have observed Slc11a2 in the apical cytoplasm of enterocytes (3). We should be able to use our Slc11a $2^{\text {int } / \text { int }}$ mice to evaluate this possibility in future experiments.

A variety of mouse strains are now available to achieve tissue-specific expression of Cre recombinase in selected cell types. By crossing additional Cre lines with Slc11a flox/flox mice, we will be able to assess the role of Slc11a2 in other tissues. While we did not find any other obvious abnormalities in Slc11a2 $12^{-/-}$mice, we cannot yet exclude other roles postulated for Slc11a2 in, for example, transfer of iron across the blood brain barrier (2) or resorption of filtered iron in the kidney $(28,29)$.

Mice heterozygous for the null Slc11a2 allele are not anemic, but they do have decreased iron stores, which suggests a mild impairment in intestinal iron transport. We are now certain that the G185R mutation found in Slc11a2 $2^{m / m k}$ mice confers partial loss of Slc11a2 function. Together, these animals represent an allelic series, intermediate between total absence of Slc11a2 and wildtype expression and function. It remains possible that a gain of calcium channel function combines with partial residual metal transporter function to ameliorate the Slc11a $2^{\mathrm{mk} / \mathrm{mk}}$ phenotype, but our results do not point to any new, independent role for altered cellular calcium homeostasis.

Consistent with earlier results (25), inactivation of Slc11a2 prevented iron overload in a mouse model of HFE hemochromatosis. Intriguingly, however, Hfe deficiency confers better survival on Slc11a2-/- animals. Although still severely anemic, compound Slc11a2 $2^{-/} \mathrm{Hfe} e^{-/-}$animals had a significantly greater red cell mass than Slc11 $a^{-/-}$animals. We do not yet understand how this occurs, but we envision 2 possibilities. First, a minor, alternate iron uptake pathway may be activated when Hfe is deficient. Alternatively, it may be that cellular iron export mechanisms are enhanced in the absence of Hfe, so that basolateral transfer of iron from enterocytes into the body is augmented and macrophage iron release is maximized. Either mechanism might increase the amount of iron delivered to erythroid precursors.

We have clear evidence for the existence of at least 1 alternate iron transport pathway functioning in the placenta and in hepatocytes. It seems likely that one or more alternative pathways also exist in other cell types, which exhaust their initial iron endowment as they divide and differentiate. This alternate transport pathway may assume greater importance in hemochromatosis, when iron overload exceeds the iron binding capacity of serum transferrin. It may explain how non-transferrin-bound iron is deposited in hepatocytes, cardiomyocytes, pancreatic acinar cells, and other sites.

What might this alternative transporter be? A calcium-dependent, non-transferrin-bound iron uptake mechanism, which also transports a variety of transition metal ions, has been described in HeLa and HepG2 cell lines $(8,11,12)$. This activity differs from that of SLC11A2, which is inhibited by calcium (2). The non-transferrin-bound iron transport system appears to require reduction of $\mathrm{Fe}^{3+}$ to $\mathrm{Fe}^{2+}$ by an extracellular ferrireductase $(10,11,30)$. Transport is active at neutral $\mathrm{pH}$, which further distinguishes it from proton-dependent SLC11A2 activity. The molecular identity of this non-transferrin-bound iron transporter remains unknown.

Recently, 2 plausible candidates for this activity have emerged. First, neutrophil gelatinase-associated lipocalin (24p3) has been shown to carry metal in the form of a siderophore-iron complex $(31,32)$. This complex binds to a specific cell surface receptor for internalization. It is not clear whether internalization is adequate for iron transfer into the cytoplasm - it may further require a transmembrane transporter, playing a role analogous to that of Slc11a2 in the transferrin cycle (33). While information to date has implicated lipocalin-mediated iron uptake only in renal development (32), it is possible that it functions elsewhere, particularly when normal uptake pathways are absent.

The second candidate is the L-type calcium channel. Mammals produce 4 nearly identical L-type calcium channels $\left(\mathrm{Ca}_{v} 1.1-1.4\right)$ with overlapping patterns of tissue expression. An L-type calcium channel has been shown to contribute to cardiac iron loading in mice (34). While these channels have typically been studied in excitable cells, they are widely expressed and might also function in hepatocyte iron uptake. We have found that an L-type calcium channel is expressed in the liver (K. Roberts and N.C. Andrews, unpublished observations). It should be possible, in future experiments, to use specific calcium channel blockers to test the possibility that an L-type calcium channel provides the alternative iron uptake route.

In summary, we have defined important roles for SLC11A2 in intestinal iron absorption and erythroid iron uptake. Our results provide direct evidence for the existence of at least 1 additional iron uptake mechanism that has physiological importance in the liver, the placenta, and possibly other tissues. Our mouse models provide new tools for identifying and studying that alternative iron transport mechanism.

\section{Methods}

Targeted disruption of the murine Slc11a2 gene. We isolated an Slc11a2 genomic clone from a strain 129 mouse bacterial artificial chromosome library (Strategene). The $3^{\prime}$ homologous region ( $3^{\prime}$-HR) of both the global 
and the conditional constructs was a $3.5 \mathrm{~kb}$ BamHI fragment from this clone inserted into the PTKLNC vector (provided by R. Mortenson, Harvard Medical School). A $3.3 \mathrm{~kb}$ BgIII/EcoR1 fragment from the genomic clone was used as the $5^{\prime}$ homologous region $\left(5^{\prime}\right.$-HR) after subcloning into pSP73 (modified by insertion of an Xhol restriction site; Promega) to provide new restriction sites. An Ncol/EcoRV fragment from the genomic clone was used as the $5^{\prime}$-HR of the conditional knockout construct after subcloning into pGEM-5zf(-) (Promega) and inserted a loxP sequence at an EcoRI site which is in intron 5 and a floxed neomycin-resistance cassette into intron 9. A BgIII/EcoRV fragment from this subclone was inserted into pSP73 (Promega) to allow excision of a $4.8 \mathrm{~kb}$ Xhol/SAII fragment containing the $5^{\prime}$-HR for the conditional knockout vector. Each targeting vector was linearized by digestion with NcoI and electroporated into $129 \mathrm{~J} 1 \mathrm{ES}$ cells. In the case of the conditional targeting construct, the neomycin-resistance cassette was subsequently excised by transient expression of Cre recombinase. Correct homologous recombination and Cre-mediated excision were confirmed by Southern blot analysis. ES cell clones with normal karyotypes were injected into C57BL/ 6 blastocysts. Transmission of the targeted alleles was confirmed by Southern blot analysis. Mice carrying the floxed Slc11a2 allele were bred with Villin-Cre mice (21) to selectively inactivate the Slc11a2 gene in the intestine. Subsequent genotyping was carried out by extracting DNA from snipped tail samples (PUREGENE kit; Gentra Systems Inc.) and subjecting it to Southern blot and/or PCR analysis. For Southern blot analysis, tail DNA was digested by BgII and probed with a PCR product generated from the 129 genomic clone using a forward primer 5'-TTTGGGGGCCCATATGCCAG-3' and a reverse primer 5'-CCCACTGCCCAGATGTAGAG-3'. For PCR analysis of the global knockout allele, we used primers TKO F1: 5'-ATGGGCGAGTTAGAGGCTTT-3' and TKO R2: 5'-TTCTCTTGGGACAATCTGGG-3'. For PCR analysis of the conditional knockout allele, we used primers TKO F1 (as above) and CKO R1: 5'-CCTGCATGTCAGAACCAATG-3'.

Animal care. All mice were born and housed in the barrier facility at Children's Hospital Boston. Female mice were analyzed unless otherwise noted. $H f e^{-/-}$and Slc11a $2^{m k / m k}$ mice used in this study were described previously $(13,35)$. We weaned pups at 28 days and maintained them on Prolab RMH 3000 LabDiet (PMI Nutrition International), which has 380 parts per million of iron. All mouse procedures were approved by the Institutional Animal Care and Use Committee at Children's Hospital Boston.

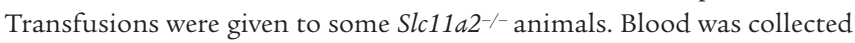
by retro-orbital bleeding of anesthetized wild-type adults using heparinized glass capillary tubes. Erythrocytes were gently pelleted and washed twice at $4{ }^{\circ} \mathrm{C}$. Prior to transfusion, packed rbcs were suspended in ice-cold PBS to a hematocrit of approximately $70 \%$. We injected $100-\mu 1 \mathrm{rbc}$ suspensions intraperitoneally into newborn pups.

Iron assays. We assayed serum iron concentrations using a serum iron/ unbound iron binding capacity kit (Thermo DMA) according to the manufacturer's instructions. We determined non-heme liver iron concentrations as previously described (36).

Enterocyte preparation and immunoblot analysis. Enterocytes were isolated from intestinal specimens as previously described (18). They were lysed in buffer containing $0.5 \%$ NP-40, $20 \mathrm{mM}$ Tris $\mathrm{pH} 8.0,100 \mathrm{mM} \mathrm{NaCl}, 40 \mathrm{mM}$ $\mathrm{NaF}, 2 \mathrm{mM}$ EDTA, $2 \mathrm{mM} \mathrm{Na}_{3} \mathrm{VO}_{4}, 2 \mathrm{mM}$ PMSF, and $10 \%$ glycerol. After centrifugation at $10,000 \mathrm{~g}$ at $4^{\circ} \mathrm{C}$ for 10 minutes, the pellet was discarded, and the supernatant fraction was analyzed. Protein concentrations were determined using the DC Protein Assay kit (Bio-Rad Laboratories). Approximately $50 \mu \mathrm{g}$ of lysate protein was mixed with sample buffer containing 2\% SDS and $100 \mathrm{mM}$ DTT, incubated at room temperature for 15 minutes, and fractionated by $12 \%$ SDS-PAGE. Electrophoresis was carried out at $120 \mathrm{~V}$ for about 80 minutes. Proteins were transferred overnight to a nitrocellulose membrane at $25 \mathrm{~mA}$. The membrane was briefly rinsed with PBS, blocked on an orbital shaker for 2 hours at room temperature with PBS containing 0.2\% Tween-20 (PBS-T) and 5\% nonfat dry milk, and then incubated for 2 more hours in the same solution containing anti-Slc11a2 antiserum $(1: 2,500)$. The membrane was washed twice for 5 minutes with PBS-T and then incubated with secondary antibody (anti-rabbit IgG-HRP, 1:4,000) in PBS-T with $2.5 \%$ milk for 2 hours. The membrane was then washed extensively with PBS-T (4 times for 15 minutes each) and then rinsed with PBS without Tween20. Enhanced chemoluminescence (ECL Plus; Amersham Biosciences) was used to develop the membrane.

Hematopoietic stem cell transplantation. We transplanted $1 \times 10^{6}$ unfractionated fetal liver cells from Slc11a2-/- and wild-type neonates (CD45.2 $\left.2^{+}\right)$into irradiated $(1,300 \mathrm{rad})(\mathrm{B} 6 . \mathrm{SJL} \times 129) \mathrm{F} 1$ recipients that were heterozygous for CD45.2 and CD45.1 alleles. Contributions from the transferred HSCs were analyzed at sacrifice after 8, 12, and 24 weeks.

Hemoglobin analysis. Hemoglobin analysis was performed as described (37). Five microliters of cystamine solution was added to $5 \mu \mathrm{l}$ of whole blood. After incubation, we performed cellulose acetate electrophoresis using Titan III plates (Helena Laboratories). Plates were stained with Ponceau S for 10 minutes and rinsed repeatedly in 5\% acetic acid to clear the background.

Flow cytometry. The contribution of donor CD $45.2^{+} \mathrm{CD} 45.1^{-}$hematopoietic cells to peripheral blood was verified by staining with biotinconjugated antibody against CD45.2 (BD Biosciences - Pharmingen) and FITC-conjugated antibody against CD45.1, followed by secondary staining with allophycocyanin-streptavidin. Cells were examined in a BD FACSCalibur machine.

Statistical analysis. Statistical significance was evaluated using the unpaired Student's $t$ test for comparisons between 2 means and analysis of variance between groups (ANOVA). Statview software version 5.0.1 (SAS Institute Inc.) was used for statistical evaluation.

\section{Acknowledgments}

This work was supported by NIH grant R01 DK53813 to N.C. Andrews, who is also an Investigator of the Howard Hughes Medical Institute. H. Gunshin was supported by an American Gastroenterological Association/Foundation for Digestive Health and Nutrition Research Scholar Award and NIH grant K01 DK02804. S. Robine was supported by the Association pour la Recherche sur le Cancer and Action Concertée Incitative Ministère de la Recherche: Biologie du développement et physiologie integrative. ES cell manipulations and blastocyst injections were carried out by the Mental Retardation Research Center Gene Manipulation Facility at Children's Hospital, funded in part by NIH grant P30 HD18655. Some blastocyst injections were carried out at the Children's Hospital Center of Excellence in Hematology core facility, funded in part by NIH grant P30 DK49216 (S. Orkin). We thank J. Jin for assistance with detection of Slc11a2 protein in enterocytes, P. Gros for his generous gift of anti-Slc11a2 antiserum, M.D. Fleming for assistance with interpretation of pathology slides, and A. Donovan for critical review of the manuscript.

Received for publication December 30, 2004, and accepted in revised form March 1, 2005.

Address correspondence to: Nancy C. Andrews, Children's Hospital Boston, Karp Family Research Laboratories RM 8-125, Boston, Massachusetts 02115-5737, USA. Phone: (617) 919-2116; Fax: (617) 432-3639; E-mail: nancy_andrews@hms.harvard.edu. 
1. Levy, J.E., Jin, O., Fujiwara, Y., Kuo, F., and Andrews, N.C. 1999. Transferrin receptor is necessary for development of erythrocytes and the nervous system. Nat. Genet. 21:396-399.

2. Gunshin, H., et al. 1997. Cloning and characterization of a mammalian proton-coupled metal-ion transporter. Nature. 388:482-488.

3. Canonne-Hergaux, F., Gruenheid, S., Ponka, P., and Gros, P. 1999. Cellular and subcellular localization of the Nramp2 iron transporter in the intestinal brush border and regulation by dietary iron. Blood. 93:4406-4417

4. Canonne-Hergaux, F., Zhang, A.S., Ponka, P., and Gros, P. 2001. Characterization of the iron trans porter DMT1 (NRAMP2/DCT1) in red blood cells of normal and anemic $\mathrm{mk} / \mathrm{mk}$ mice. Blood. 98:3823-3830.

5. Trinder, D., Oates, P.S., Thomas, C., Sadleir, J., and Morgan, E.H. 2000. Localisation of divalent metal transporter 1 (DMT1) to the microvillus membrane of rat duodenal enterocytes in iron deficiency, but to hepatocytes in iron overload. Gut. 46:270-276.

6. Gambling, L., et al. 2001. Effect of iron deficiency on placental transfer of iron and expression of iron transport proteins in vivo and in vitro. Biochem. $J$. 356:883-889.

7. Georgieff, M.K., Wobken, J.K., Welle, J., Burdo, J.R., and Connor, J.R. 2000. Identification and localization of divalent metal transporter-1 (DMT-1) in term human placenta. Placenta. 21:799-804

8. Inman, R.S., and Wessling-Resnick, M. 1993. Characterization of transferrin-independent iron transport in $\mathrm{K} 562$ cells. Unique properties provide evidence for multiple pathways of iron uptake. J. Biol. Chem. 268:8521-8528.

9. Jordan, I., and Kaplan, J. 1994. The mammalian transferrin-independent iron transport system may involve a surface ferrireductase activity. Biochem. J. 302:875-879.

10. Kaplan, J., Jordan, I., and Sturrock, A. 1991. Regulation of the transferrin-independent iron transport system in cultured cells. J. Biol. Chem. 266:2997-3004.

11. Randell, E.W., Parkes, J.G., Olivieri, N.F., and Templeton, D.M. 1994. Uptake of non-transferrin bound iron by both reductive and non-reductive processes is modulated by intracellular iron. J. Biol. Chem. 269:16046-16053.

12. Sturrock, A., Alexander, J., Lamb, J., Craven, C.M., and Kaplan, J. 1990. Characterization of a transferrin-independent uptake system for iron in HeLa cells. J. Biol. Chem. 265:3139-3145.

13. Fleming, M.D., et al. 1998. Nramp2 is mutated in the anemic Belgrade (b) rat: evidence of a role for Nramp2 in endosomal iron transport. Proc. Natl. Acad. Sci. U. S. A. 95:1148-1153.

14. Fleming, M.D., et al. 1997. Microcytic anemia mice have a mutation in Nramp2, a candidate iron transporter gene. Nat. Genet. 16:383-386.

15. Canonne-Hergaux, F., et al. 2000. The Nramp2/ DMT1 iron transporter is induced in the duodenum of microcytic anemia mk mice but is not properly targeted to the intestinal brush border. Blood. 96:3964-3970.

16. Su, M.A., Trenor, C.C., Fleming, J.C., Fleming, M.D., and Andrews, N.C. 1998. The G185R mutation disrupts function of iron transporter Nramp2. Blood. 92:2157-2163.

17. Touret, N., et al. 2004. Molecular and cellular mechanisms underlying iron transport deficiency in microcytic anemia. Blood. 104:1526-1533.

18. Xu, H., Jin, J., DeFelice, L.J., Andrews, N.C., and Clapham, D.E. 2004. A spontaneous, recurrent mutation in divalent metal transporter-1 exposes a calcium entry pathway. PLoS Biol. 2:E50. doi:10.1371/journal.pbio.0020050.

19. Mims, M.P., et al. 2005. Identification of a human mutation of DMT1 in a patient with microcytic anemia and iron overload. Blood. 105:1337-1342.

20. Andrews, N.C. 2000. Iron homeostasis: insights from genetics and animal models. Nat. Rev. Genet. 1:208-217.

21. el Marjou, F., et al. 2004. Tissue-specific and inducible Cre-mediated recombination in the gut epithelium. Genesis. 39:186-193.

22. Canonne-Hergaux, F., et al. 2001. Expression of the DMT1 (NRAMP2) iron transporter in mice with genetic iron overload disorders. Blood. 97:1138-1140.

23. Russell, E.S., et al. 1970. Characterization and genetic studies of microcytic anemia in house mouse. Blood. 35:838-850
24. Russell, E.S., McFarland, E.C., and Kent, E.L. 1970. Low viability, skin lesions, and reduced fertility associated with microcytic anemia in the mouse. Transplant. Proc. 2:144-151.

25. Levy, J.E., Montross, L.K., and Andrews, N.C. 2000 Genes that modify the hemochromatosis phenotype in mice. J. Clin. Invest. 105:1209-1216.

26. Ned, R.M., Swat, W., and Andrews, N.C. 2003. Transferrin receptor 1 is differentially required in lymphocyte development. Blood. 102:3711-3718.

27. Tenhunen, R., Grasbeck, R., Kouvonen, I., and Lundberg, M. 1980. An intestinal receptor for heme: its parital characterization. Int. J. Biochem. 12:713-716.

28. Canonne-Hergaux, F., and Gros, P. 2002. Expression of the iron transporter DMT1 in kidney from normal and anemic mk mice. Kidney Int. 62:147-156.

29. Ferguson, C.J., et al. 2001. Cellular localization of divalent metal transporter DMT-1 in rat kidney. Am. J. Physiol. Renal Physiol. 280:F803-F814.

30. Inman, R.S., Coughlan, M.M., and Wessling-Resnick, M. 1994. Extracellular ferrireductase activity of $\mathrm{K} 562$ cells is coupled to transferrin-independent iron transport. Biochemistry. 33:11850-11857.

31. Goetz, D.H., et al. 2002. The neutrophil lipocalin NGAL is a bacteriostatic agent that interferes with siderophore-mediated iron acquisition. Mol. Cell. 10:1033-1043.

32. Yang, J., et al. 2002. An iron delivery pathway mediated by a lipocalin. Mol. Cell. 10:1045-1056.

33. Kaplan, J. 2002. Mechanisms of cellular iron acquisition: another iron in the fire. Cell. 111:603-606.

34. Oudit, G.Y., et al. 2003. L-type $\mathrm{Ca}(2+)$ channels provide a major pathway for iron entry into cardiomyocytes in iron-overload cardiomyopathy. Nat. Med. 9:1187-1194.

35. Levy, J.E., Montross, L.K., Cohen, D.E., Fleming, M.D., and Andrews, N.C. 1999. The C282Y mutation causing hereditary hemochromatosis does not produce a null allele. Blood. 94:9-11.

36. Torrance, J.D., and Bothwell, T.H. 1980. Tissue iron stores. Churchill Livingstone. New York, New York, USA.

37. Whitney, J.B., III. 1978. Simplified typing of mouse hemoglobin ( $\mathrm{Hbb})$ phenotypes using cystamine. Biochem. Genet. 16:667-672. 